\title{
Article \\ Antibiotics to Prevent Surgical Site Infection (SSI) in Oral Surgery: Survey among Italian Dentists
}

\author{
Marco Lollobrigida *(D), Gianluca Pingitore, Luca Lamazza (D), Giulia Mazzucchi, Giorgio Serafini \\ and Alberto De Biase
}

Citation: Lollobrigida, M.; Pingitore, G.; Lamazza, L.; Mazzucchi, G.; Serafini, G.; De Biase, A. Antibiotics to Prevent Surgical Site Infection (SSI) in Oral Surgery: Survey among Italian Dentists. Antibiotics 2021, 10, 949. https://doi.org/10.3390/ antibiotics10080949

Academic Editor: Thomas E. Rams

Received: 30 June 2021

Accepted: 4 August 2021

Published: 6 August 2021

Publisher's Note: MDPI stays neutral with regard to jurisdictional claims in published maps and institutional affiliations.

Copyright: (C) 2021 by the authors. Licensee MDPI, Basel, Switzerland. This article is an open access article distributed under the terms and conditions of the Creative Commons Attribution (CC BY) license (https:/ / creativecommons.org/licenses/by/ $4.0 /)$.
Department of Oral and Maxillofacial Sciences, Sapienza University of Rome, 00161 Rome, Italy; pingitore.1631372@studenti.uniroma1.it (G.P.); luca.lamazza@uniroma1.it (L.L.); mazzucchi.1536500@studenti.uniroma1.it (G.M.); giorgio.serafini@uniroma1.it (G.S.); alberto.debiase@uniroma1.it (A.D.B.)

* Correspondence: marco.lollobrigida@uniroma1.it; Tel.: +39-06-4997-6626

Abstract: The benefit of an antibiotic prophylaxis for most oral surgical procedures is controversial. The aim of this study was to collect information on the prescribing habits of a sample of Italian dentists with respect to the role of antibiotic prophylaxis in preventing surgical site infections (SSI). An anonymous questionnaire was prepared and made accessible online by sharing a Google Forms link. General anagraphic data and educational background information were collected to obtain a profile of the participants. Different clinical scenarios were then proposed, with the participants asked to choose whether they would prescribe an antibiotic prophylaxis and with which dosage regimens. In total, 169 dentists participated in the questionnaire and the obtained data were assessed through a percentage report. The results showed a substantial agreement in antibiotics prescription, but only in a limited number of clinical scenarios, such as deciduous teeth extraction or simple extractions in healthy adult patients. Discordant responses were found for several clinical cases, particularly for cases of comorbidities, surgical or multiple extractions, implant placement and abscess drainage. The answers obtained from the survey sample were notably heterogeneous, indicating that the choice to prescribe an antibiotic prophylaxis to prevent SSIs is often discretionary. Moreover, the dosage regimen of prophylaxis is also controversial. The results of this study demonstrate the need for specific guidelines on antibiotics in dentistry and, specifically, on antibiotic prophylaxis in oral surgery. Such guidelines would help to avoid unnecessary prescriptions.

Keywords: antibiotics; antibiotic prophylaxis; surgical site infection; postoperative infection; oral surgery; antimicrobial resistance; antibiotic resistance

\section{Introduction}

Antibiotics are among the most common medications prescribed by dentists, both as a therapy of odontogenic infections and as prophylaxis to prevent surgical site infections (SSI) [1]. Although antibiotic therapies are dictated by objective infectious diseases, there are no specific guidelines concerning the use of antibiotics to prevent SSIs following oral surgical procedures. Therefore, the prescription of antibiotic prophylaxis (AP) is often left to the personal experience and considerations of dental clinicians. More often, antibiotics are prescribed either as standard practice for any kind of oral surgical procedure [2], or as a form of defensive medicine [3]. This leads to a general overprescription and contributes to an increased risk of antimicrobial resistance (AMR) in the population.

Perioperative antibiotic prophylaxis is defined as the administration of antibiotics before or during a surgical procedure to prevent SSI episodes [4]. Some authors distinguish between primary prophylaxis, secondary prophylaxis and eradication. Primary prophylaxis is defined as the prevention of an initial infection; secondary prophylaxis is defined as the prevention of the recurrence or reactivation of a pre-existing infection; and eradication refers to the elimination of a colonized organism to prevent the development 
of an infection [5]. According to these case definitions, most APs in dentistry consist of primary or secondary prophylaxis.

Although the European Centre for Disease Prevention and Control (ECDC) did not address oral and maxillofacial surgery in its report [4], the guidelines developed by the American Society of Health-System Pharmacists (ASHP), the Infectious Diseases Society of America (IDSA), the Surgical Infection Society (SIS), and the Society for Healthcare Epidemiology of America (SHEA) dedicate one section to head and neck surgery, though this is not specific to the dental field [5]. According to these guidelines, a head and neck procedure involving the incision of the oral or pharyngeal mucosa is to be considered as clean-contaminated; thus, an AP is recommended for most of these procedures. However, oral surgical procedures are less invasive and, even in clean-contaminated conditions, do not usually require antibiotic prophylaxis.

Different factors can be related to an increased risk of SSI, including procedure- and patient-related factors. The former comprises the duration and invasiveness of the procedure, bone instrumentation, the use of biomaterials, the surgical technique and sterilization practices. Patient-related factors include age, nutritional status, diabetes, smoking, obesity, coexisting infections or contaminations, and altered immune responses [6]. As such, clinicians may be disoriented by the number of surgical procedures and conditions that may require an AP. As a general principle, the prescription of prophylactic antibiotics should be reasonable and weighted on the basis of a risk-benefit analysis, with consideration also given to the consequences of unnecessary administrations on increases in AMR among the general population [7]. The last annual national report of the Italian drug agency [8] painted a worrying picture of AMR, noting how the high consumption of antibiotics is increasing the spread of AMR, and estimating that antibiotics are over-prescribed more than $25 \%$ of the time. Another study on elective minor surgical procedures found that in approximately half of all cases, the antibiotic prophylaxis was not consistent with the guidelines [9]. No specific data have been reported, however, about the use of antibiotics in dentistry and, more importantly, on the specific indication for prescriptions, distinguishing between AP for a cardiac condition, AP to prevent SSI, and therapeutic prescription [10]. For this reason, it is important to investigate the prescribing habits of dentists and to assess whether antibiotic prescriptions are inappropriate with respect to current evidences. By comparing results from other national surveys, these data may provide the basis for elaborating international guidelines and antibiotic stewardship programs on the correct use of AP to prevent SSIs in dentistry.

The objective of this study was to collect information on the prescribing habits of a sample of Italian dentists with respect to antibiotic prophylaxis for the most common oral surgical procedures.

\section{Results}

At the survey's conclusion, 169 replies were collected with a response rate of $6.76 \%$. The modality of the questionnaire administration did not allow the characteristics of nonrespondents to be disclosed. Demographic data of respondents are reported in Table 1. The age of respondents ranged from 24 to 69 years, with a median age of 40 years. Most respondents were male $(71 \%)$, with a Degree in Dentistry $(82 \%)$ and professional experience of at least 5 years $(80.5 \%)$. The most frequent specialization was Oral Surgery, though $69.2 \%$ of respondents had no specialization. Most replies came from Central Italy and from private practitioners (60.9). These data indicate that the respondents, in terms of their characteristics, can be considered representative of the general population.

A graphical representation of the answers to both the clinical cases and the shortform considerations are reported in Figure 1 and Table 2, respectively. Overall, only in a few cases was a strong agreement found among participants, with the responses varying considerably for most questions. 
Table 1. Demographic data of participants.

\begin{tabular}{cccc}
\hline & & $\mathbf{N}^{\circ}$ & $\%$ \\
\hline \multirow{2}{*}{ Gender } & Male & 120 & 71 \\
& Female & 49 & 29 \\
\hline \multirow{2}{*}{ Educational } & Degree in Dentistry & 140 & 82.84 \\
qualification & Degree in Medicine and Surgery & 20 & 11.83 \\
& specialized in Odontostomatology & 9 & 5.33 \\
\hline \multirow{3}{*}{ Years of professional } & Degree in Medicine and Surgery & 33 & 19.53 \\
experience & $<5$ & 46 & 27.22 \\
& $5-10$ & 13 & 7.69 \\
& $10-15$ & 77 & 45.56 \\
\hline \multirow{3}{*}{ Dental speciality } & $>15$ & 43 & 25.44 \\
& Oral surgery & 8 & 4.73 \\
& Orthodontics & 4 & 2.37 \\
& Pediatric Dentistry & 117 & 69.23 \\
\hline \multirow{2}{*}{ Region } & None & 40 & 23.67 \\
& Northern Italy & 95 & 56.21 \\
& Central Italy & 34 & 20.12 \\
\hline \multirow{2}{*}{ Employment } & Southern and Insular Italy & 103 & 60.95 \\
& Private practice & 12 & 7.1 \\
\hline
\end{tabular}

Table 2. Responses to questions about the use of antibiotics in specific clinical scenarios and antibiotic resistance awareness. Percentages are rounded to the first decimal place. Questions are reported in Figure 2.

\begin{tabular}{|c|c|c|}
\hline Question & Answer & $\%$ \\
\hline \multirow{4}{*}{$\mathrm{C} 1$} & Always, even with no visible plaque and calculus deposits & 27.8 \\
\hline & Only in case of abundant plaque and calculus deposits & 57.4 \\
\hline & Not always, even in case of abundant plaque and calculus deposits & 14.2 \\
\hline & Never & 0.6 \\
\hline \multirow{2}{*}{$\mathrm{C} 2$} & Yes & 57.4 \\
\hline & No & 42.6 \\
\hline \multirow{2}{*}{ C3 } & Yes & 17.8 \\
\hline & No & 82.2 \\
\hline \multirow{2}{*}{$\mathrm{C} 4$} & Yes & 83.4 \\
\hline & No & 16.6 \\
\hline \multirow{2}{*}{ C5 } & Yes & 42.6 \\
\hline & No & 57.4 \\
\hline \multirow{2}{*}{ C6 } & Yes & 54.4 \\
\hline & No & 45.6 \\
\hline \multirow{2}{*}{$\mathrm{C} 8$} & Yes & 74.6 \\
\hline & No & 25.4 \\
\hline \multirow{3}{*}{ C9 } & Yes, I feel very informed & 26.6 \\
\hline & I am quite informed but I feel the need to further master this issue & 66.9 \\
\hline & I think I am not adequately informed & 6.5 \\
\hline \multirow{4}{*}{$\mathrm{C} 10$} & Not so widespread & 5.3 \\
\hline & Medium widespread & 26 \\
\hline & Very widespread & 47.3 \\
\hline & Extremely widespread & 21.3 \\
\hline
\end{tabular}


Q1

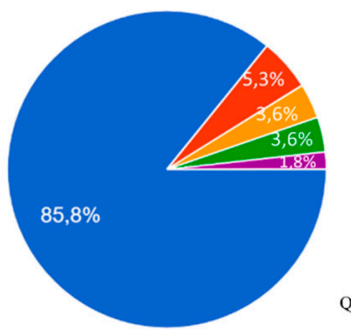

Q4

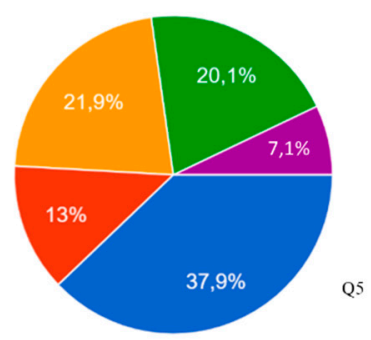

Q7

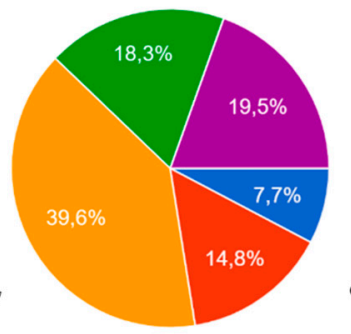

Q8
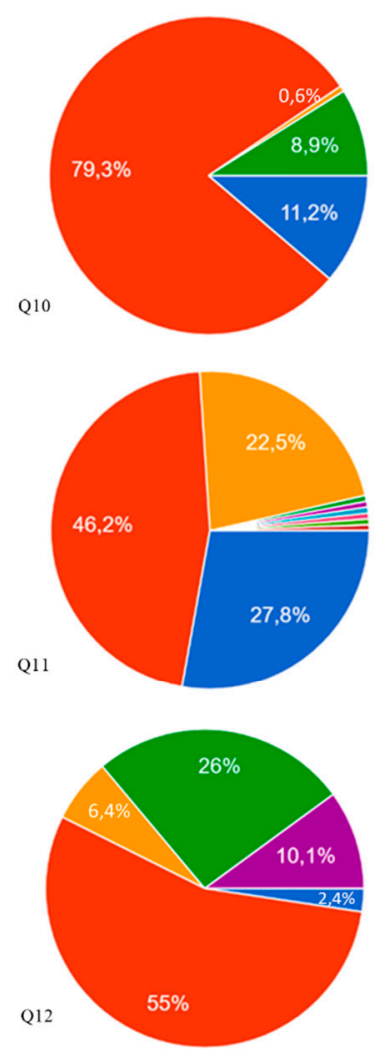
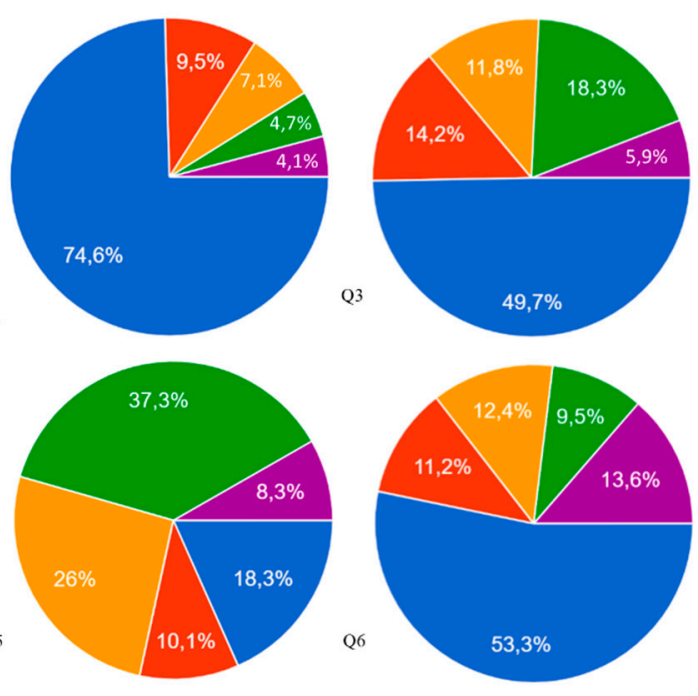

- No antibiotic therapy

Broad-spectrum antibiotic in a single dose 1 hour before surgery

Broad-spectrum antibiotic 1 hour before surgery + post-operative for 5 days

- Broad-spectrum antibiotic from $24 / 48$ hours

before surgery + post-operative for $3 / 4$ days

- Broad-spectrum antibiotic for $5 / 7$ days after surgery

- Broad-spectrum antibiotic for 5 days and anti-inflammatory/analgesic drugs as needed

- Local antiseptics (chlorhexidine), anti-inflammatory/analgesic drugs and oral hygiene instructions, if after 3 days there is no remission of the symptoms, then I prescribe a
broad-spectrum antibiotic for 5 days

- I wait for spontaneous remission of symptoms; if after 3 days there is no remission of the symptoms, then I prescribe a broad-spectrum antibiotic for 5 days

- Immediate surgical extraction of the tooth and post-operative broad-spectrum antibiotic for 5 days

- Abscess drainage from the pocket through manual and ultrasonic scaling

- Abscess drainage from the pocket through manual and ultrasonic scaling followed by broad-spectrum antibiotic for 5 days

- Broad-spectrum antibiotic prescription for 5 days followed by manual and ultrasonic scaling

+ Free answer

- Local antiseptics $(0.2 \%$ chlorhexidine $)$ and broad-spectrum antibiotic therapy in a single dose hour before surgery

- Local antiseptics $(0.2 \%$ chlorhexidine) and polvpharmacological antibiotic therapy (amoxicillin metronidazole) pre-operative $2 / 3$ days before surgery + post-operative for $5 / 7$ days

- Local antiseptics ( $0.2 \%$ chlorhexidine) and post-operative polypharmacological antibiotic therapy (amoxicillin + metronidazole) for $5 / 7$ day

- Local antiseptics $(0.2 \%$ chlorhexidine) and polvpharmacological antibiotic therapy (amoxicillin metronidazole) pre-operative from 1 day before surgery + post-operative for $5 / 7$ days

- The patient is sent to a specialized hospital

Figure 1. Graphs summarizing responses to specific questions about the use of prophylactic antibiotics to prevent SSI after the most common oral surgical procedures. Percentages are rounded to the first decimal place. The questions are reported in Figure 2. 
Q1- Non-surgical extraction by odontotomy of an infraoccluded primary molar in an 11-year-old patient. Negative history for systemic diseases.

a) No antibiotic therapy

b) Broad-spectrum antibiotic in a single dose 1 hour before surger

c) Broad-spectrum antibiotic 1 hour before surgery + post-operative for 5 days

d) Broad-spectrum antibiotic from $24 / 48$ hours before surgery + post-operative for $3 / 4$ days

e) Broad-spectrum antibiotic for $5 / 7$ days after surgery

Q2 - Non-surgical extraction of 2.4 with destructive caries in a 56-year-old patient Absence of acute and/or chronic periapical inflammatory lesions. Arterial hypertension under treatment.

a) No antibiotic therapy

b) Broad-spectrum antibiotic in a single dose 1 hour before surgery

c) Broad-spectrum antibiotic 1 hour before surgery + post-operative for 5 days

d) Broad-spectrum antibiotic from $24 / 48$ hours before surgery + post-operative for $3 / 4$ days

e) Broad-spectrum antibiotic for $5 / 7$ days after surgery

Q3 - Non-surgical extraction of 2.6 with mobility of grade 3 in 88-year-old patient. ASA II, antiplatelet herapy, arterial hypertension under treatment

a) No antibiotic therapy

Broad-spectrum antibiotic in a single dose 1 hour before surgery

c) Broad-spectrum antibiotic 1 hour before surgery + post-operative for 5 days

d) Broad-spectrum antibiotic from $24 / 48$ hours before surgery + post-operative for $3 / 4$ days

e) Broad-spectrum antibiotic for $5 / 7$ days after surgery

Q4 - Non-surgical extraction of 3.4 and 3.5 in a 75 -year-old patient. Absence of acute and/or chronic

periapical inflammatory lesions. Arterial hypertension under treatment and well-controlled type 2 diabetes. a) No antibiotic therapy

b) Broad-spectrum antibiotic in a single dose 1 hour before surger

c) Broad-spectrum antibiotic 1 hour before surgery + post-operative for 5 days

d) Broad-spectrum antibiotic from $24 / 48$ hours before surgery + post-operative for $3 / 4$ days

e) Broad-spectrum antibiotic for $5 / 7$ days after surgen Q5 - Non-surgical extraction of three root remnants of monoradicular teeth in a 57 -year-old patient. At $x$-ray
two root remnants present periapical granuloma. History of myocardial infarction, dyslipidemia, BMI> 35 , two root remnants present periapical granuloma. History

a) No antibiotic therapy

Broad-spectrum antibiotic in a single dose 1 hour before surgery

Broad-spectrum antibiotic 1 hour before surgery + post-operative for 5 days

d) Broad-spectrum antibiotic from $24 / 48$ hours before surgery + post-operative for $3 / 4$ days

e) Broad-spectrum antibiotic for $5 / 7$ days after surgery

Q6 - Non-surgical extraction of five root remnants $(1.5,1.3,2.2,2.4,2.6)$ in a 68 -year-old patient. Absence of cute and/or chronic periapical inflammatory lesions. Negative history for systemic diseases.

a) No antibiotic therapy

Broad-spectrum antibiotic in a single dose 1 hour before surgery

C) Broad-spectrum antibiotic 1 hour before surgery + post-operative for 5 days

Broad-spectrum antibiotic from $24 / 48$ hours before surgery + post-operative for $3 / 4$ days

e) Broad-spectrum antibiotic for $5 / 7$ days after surgery

Q7 - Surgical extraction of 4.8 in complete bone impaction in a 25 -year-old patient. Negative history for systemic diseases.

a) No antibiotic therapy

Broad-spectrum antibiotic in a single dose 1 hour before surgery

Broad-spectrum antibiotic 1 hour before surgery + post-operative for 5 days

Broad-spectrum antibiotic from 24/48 hours before surgery + post-operative for $3 / 4$ days

Broad-spectrum antibiotic for $5 / 7$ days after surgery

Q8 - Insertion of one endosseous implant in the mandibular molar region with conventional technique lopen flap) in a 54-year-old patient, Negative history for systemic diseases.

a) No antibiotic therapy

b) Broad-spectrum antibiotic in a single dose 1 hour before surgery

c) Broad-spectrum antibiotic 1 hour before surgery + post-operative for 5 days

d) Broad-spectrum antibiotic from $24 / 48$ hours before surgery + post-operative for $3 / 4$ days

e) Broad-spectrum antibiotic for $5 / 7$ days after surgery

Q9- Non-surgical extraction of 1.4 and 1.5 in 55 -year-old patient with metabolic syndrome. Absence of acute and/or chronic periapical inflammatory lesions. Pre-operative blood glucose: $160 \mathrm{mg} / \mathrm{dL}$.

a) No antibiotic therapy

b) Broad-spectrum antibiotic in a single dose 1 hour before surger

c) Broad-spectrum antibiotic 1 hour before surgery + post-operative for 5 days

d) Broad-spectrum antibiotic from 24/48 hours before surgery + post-operative for 3/4 days

e) Broad-spectrum antibiotic for $5 / 7$ days after surgery

Q10 - 17-year-old patient reports continuous mild pain for 2 days at the level of 3.8 in partial osteo-mucosal impaction. No fever. Upon physical examination, the pericoronal tissues are erythematous and edematous. without purulent exudate.

a) Broad-spectrum antibiotic for 5 days and anti-inflammatory/analgesic drues as needed

b) Local antiseptics (chlorhexidine), anti-inflammatory/analgesic drugs and oral hygiene instructions: if after 3 days there is no remission of the symptoms, then I prescribe a broad spectrum antibiotic for 5 days

c) I wait for spontaneous remission of symptoms; if after 3 days there is no remission of the

symptoms, then I prescribe a broad-spectrum antibiotic for 5 days

d) Immediate surgical extraction of the tooth and post-operative broad-spectrum antibiotic for 5 days
Q11-54-year-old male patient presenting with a fluctuating swelling, of about $7 \mathrm{~mm}$ diameter, on the buccal gingiva of 2.6. No dental mobility. Presence of sulcular suppuration upon palpation; vestibular probing depth three sites: 9-8-9 mm; no fever, no lymphadenopathy.

a) Abscess drainage from the pocket through manual and ultrasonic scaling

b) Abscess drainage from the pocket through manual and ultrasonic scaling followed by broadspectrum antibiotic for 5 days

c) Broad-spectrum antibiotic prescription for 5 days followed by manual and ultrasonic scaling d) Other (short answer)

Q12 - Non-surgical extraction of 3.5 with coronal-root fracture in a 62 -year-old patient. Osteoporosis treated for 11 years with bisphosphonate (sodium alendronate by mouth, $70 \mathrm{mg}$ once a week) suspended for 30 days. Negative history for other systemic diseases.

a) Local antiseptics ( $0.2 \%$ chlorhexidine) and broad-spectrum antibiotic therapy in a single dose 1 hour before surgery

b) Local antiseptics ( $0.2 \%$ chlorhexidine) and polypharmacological antibiotic therapy (amoxicillin + metronidazole) pre-operative $2 / 3$ days before surgery + post-operative for $5 / 7$ days

c) Local antiseptics ( $0.2 \%$ chlorhexidine) and post-operative polypharmacological antibiotic therapy (amoxicillin + metronidazole) for $5 / 7$ days

d) Local antiseptics ( $0.2 \%$ chlorhexidine) and polypharmacological antibiotic therapy (amoxicillin + metronidazole) pre-operative from 1 day before surgery + post-operative for $5 / 7$ days

e) I refer the patient to a specialized hospita

C1 - Do you perform a professional oral hygiene session before non-surgical extractions?

a) Always, even with no visible plaque and calculus deposits.

b) Only in case of abundant plaque and calculus deposits

c) Not always, even in case of abundant plaque and calculus deposits

d) Never

C2 - "Poor oral hygiene and low patient compliance may indicate postoperative antibiotic coverage". Do you agree with this statement?

a) Yes

c3 - "The insertion of haemostatic materials (fibrin sponges, collagen, etc.) in the post-extraction socket and the application of sutures always require postoperative antibiotic coverage". Do you agree with this statement?

a) Yes

C4 - Do you think post-operative antibiotic coverage is useful in case of ridge preservation procedures by grafting bone substitutes in the post-extraction socket:

a) Yes

C5 - Do you consider useful post-operative antibiotic coverage in case of non-surgical extractions of teeth with periapical granuloma?

$$
\begin{array}{ll}
\text { a) Yes } \\
\text { b) No }
\end{array}
$$

C6 - Have you ever prescribed an antibiotic prophylaxis that you did not consider strictly necessary? a) Yes
b) No

C7 - If you answered "Yes" to the previous question, what reasons led you to prescribe the antibiotic anyway (select one or more answers)

a) Request/insistence by the patient
b) Persistent post-operative pain
c) To avoid possible disputes or complaints by the patient
d) Impossibility to visit the patient
e) To perform an exclusion diagnosis
f) Apprehensive patient
g) Other

CB-Have you attended refresher courses and/or read scientific papers specifically on the correct use of antibiotics in dentistry in the last 2 years? a) res

99- Are you informed about the antibiotic resistance phenomenon and the antibiotic prescribing appropriateness?

b) I am quite informed, but I feel the need to further master this issue

c) I think I am not adequately informed

C10 - How widespread do you think is the problem of inappropriate antibiotic prescription in the dental

field?

a) Not so widespread
b) Medium widespread
c) Very widespread
d) Extremely widespread

Figure 2. Questionnaire including clinical cases (Q1-Q12) and short-form considerations (C1-C10) about the use of antibiotics in specific dental procedures. 
A moderate agreement on not prescribing antibiotics was found in Q1 (85.9\%) and Q2 (74.6\%), which concerned the extraction of a deciduous molar and a simple premolar extraction, respectively, in a 56-year-old patient with arterial hypertension.

The percentage of "no antibiotic" response decreased in Q3 (49.7\%), Q4 (37.9\%), Q5 (18.3\%) and Q6 (53.3\%), which concerned cases of non-surgical single or multiple extractions in patients with well-controlled systemic diseases, and in Q9 (26\%), where the patient had a perioperative uncontrolled blood glucose (BG). Moreover, an agreement was not found on timing and posology.

In Q7 (the surgical extraction of an impacted wisdom tooth), only $7.7 \%$ of participants would not prescribe antibiotics or would prescribe a single preoperative dose $(14.8 \%)$, whereas $77.4 \%$ of respondents would prescribe pre- and post-operative antibiotics or exclusively postoperative antibiotics.

As for Q8 (implant surgery), responses were also heterogeneous, with only 20.1\% of clinicians prescribing exclusively one preoperative dose, and $14.8 \%$ not prescribing any antibiotic. The remaining respondents opted for postoperative AP with or without preoperative doses.

In Q10 (the pericoronitis case), 79.3\% of the participants chose to use local antiseptics and anti-inflammatories and to re-evaluate after 3 days, avoiding antibiotics in the first instance. On the contrary, in Q11, the majority of respondents (46.2\%) agreed in prescribing a 5-day antibiotic therapy following drainage and manual/ultrasonic debridement of the pocket; $22.5 \%$ would prescribe antibiotics before scaling, whereas only $27.8 \%$ would perform drainage and scaling without antibiotics.

In Q12, most respondents opted for perioperative AP starting from 2 to 3 days (55\%) or 1 day (26\%) before the extraction; $6.4 \%$ would prescribe only postoperative AP, $2.4 \%$ would prescribe only a single preoperative dose, and $10.1 \%$ would send the patient to a specialized hospital.

In the third section, short-form considerations were proposed on specific issues. $\mathrm{C} 1$ and $\mathrm{C} 2$ were meant to assess the relationship between oral hygiene conditions and antibiotic prescriptions. In C1, clinicians were asked whether they performed an oral hygiene session (OHS) before non-surgical extraction: $57.4 \%$ performed an OHS only in cases of abundant plaque and calculus deposits, $27.8 \%$ always performed an OHS, $14.2 \%$ did not always perform an OHS even in cases of scarce oral hygiene, and $0.6 \%$ never performed an OHS before non-surgical extractions. In $\mathrm{C} 2,57.4 \%$ of respondents considered poor oral hygiene and low patient compliance an indication for AP. On the contrary, $82.2 \%$ of respondents did not consider the use of haemostatic materials in post-extractive sockets to be an indication for the prescription of antibiotics (C3), whereas $83.4 \%$ prescribed postoperative AP in the case of a ridge preservation procedure with bone substitutes (C4). In C5, 42.6\% of respondents considered postoperative antibiotics useful after the non-surgical extraction of teeth with periapical granuloma. More than half of respondents $(54.4 \%)$ referred to having prescribed AP despite considering it unnecessary (C6-C7). The principal reasons for unnecessary prescriptions included concerns regarding disputes or complaints from the patients $(44.7 \%)$, post-operative pain management $(39.4 \%)$, patient's request $(34 \%)$, impossibility to visit the patient $(26.6 \%)$, and apprehensive patients (18.1\%). Interestingly, in the free answers, some reported insistence by other clinicians.

Finally, $74.6 \%$ of respondents referred to having attended courses or having read scientific papers on the use of antibiotics in dentistry during the last two years (C8). However, only $26.6 \%$ of respondents considered themselves fully informed on the issue of AMR and prescription appropriateness, with $66.9 \%$ considering themselves informed enough, but in need of more information (C9). Significantly, most respondents considered inappropriate antibiotic prescriptions as medium (26\%), very $(47.3 \%)$ or extremely $(21.3 \%)$ widespread (C10). 


\section{Discussion}

Antibiotics represent an inestimable resource, helping to save the lives of millions worldwide from potentially fatal infectious diseases. Several guidelines and programs have been developed to prevent AMR phenomena by reducing the inappropriate use of antibiotics. Notwithstanding, inappropriate prescriptions are still widespread [11]. In this context, the results of this study indicate that prophylactic administrations of antibiotics in dentistry are mainly based on subjective considerations, and only occasionally does an agreement exist on indications, dosage and timing.

A general agreement was found in not prescribing AP after non-surgical extractions in healthy patients, whereas different approaches were followed for patients with comorbidities. There are few studies on the efficacy of AP for the extraction of normally erupted teeth. In a recent retrospective study [12] on 418 patients who underwent non-surgical extractions, 280 received antibiotics, whereas 138 did not receive any medication. Only 12 total cases of alveolitis were reported $(2.87 \%)$, with half of those cases receiving antibiotics. It was concluded that antibiotics do not prevent post-extractive alveolitis after the extraction of erupted teeth. These observations are supported by an RCT [13], which confirmed that antibiotics are not effective in the prevention of SSI after non-surgical extractions. It is important to note that, with the exception of cardiac conditions at the highest risk of infective endocarditis [14,15], common cardiovascular conditions such as arterial hypertension are not an indication for AP [16]. Moreover, there are currently no evident correlations between old age and an increased risk of post-extractive infections [12].

In the case of an extraction of a lower third molar with complete bony impaction, the vast majority of respondents $(77.4 \%)$ would prescribe pre- and post-operative or only postoperative antibiotics, whereas only $7.7 \%$ of participants would not prescribe antibiotics or would prescribe a single preoperative dose $(14.8 \%)$. The use of antibiotics after surgical extractions is still controversial. In a randomized, double-blind, placebo-controlled trial including 118 patients undergoing the surgical extraction of impacted third molars, Arteagoitia et al. [17] found no significant differences in SSI incidence between the treated group ( $2 \mathrm{~g}$ amoxicillin/125 $\mathrm{mg}$ clavulanic acid $2 \mathrm{~h}$ before the surgery and postoperatively twice a day for 4 days) and the placebo group. These data are supported by the observation that the risk of infection related to the extraction of impacted lower third molars is, in general, lower than $6 \%$ [18], leading some authors to discourage the routine use of antibiotics in healthy patients in the absence of pre-existing infections or complicated and long-lasting procedures. On the contrary, a recent Cochrane systematic review [19] reported a lower incidence of postsurgical infectious complications after third molar extractions in patients treated with antibiotics, compared to a placebo. In 21 of the 23 reviewed studies, only impacted third molars were included. According to the authors, antibiotics may reduce the risk of infection by approximately $66 \%$, although this is with a low-certainty evidence and no definitive conclusion about the best timing of administration. Nevertheless, as already noted by Sancho-Puchades [20], it is often difficult to distinguish between infectious and non-infectious complications, because pain, acute swelling and trismus may be secondary both to the surgical trauma or to infections. It should, therefore, be considered a possible diagnostic bias among different studies due to the presence of common postoperative complications misdiagnosed as postoperative infections.

As for implant surgery, different studies have indicated that a single preoperative dose of a wide-spectrum antibiotic can reduce early implant loss (1.8\% vs. 5.6\%) [21] but does not prevent SSI [21-23]. Only $20.1 \%$ of the participants opted for a single preoperative dose; similar findings have been reported in a previous survey [24]. Cross-sectional studies conducted in other countries have shown that an exclusively preoperative prophylaxis was prescribed by $32.4 \%$ of a Dutch sample [25] and 5.73\% of a Spanish sample [26]. Based on the current evidence, postoperative antibiotics may not be shown to prevent early implant failures and SSIs in healthy patients undergoing implant surgery [26]. Furthermore, studies and reviews suggest that in the case of "straightforward" implant surgery in healthy patients, antibiotics should not be prescribed $[21,27,28]$. However, despite this, a review 
of epidemiological investigations showed that three quarters of participants routinely prescribe antibiotics in implant surgery procedures in healthy patients [29].

Diabetes mellitus is commonly considered an independent risk factor for SSIs following surgical procedures [30]. Despite the paucity of good evidence in the dental field, most clinicians assume a higher risk for postoperative infections in diabetic patients. This putative excess risk is followed by recommendations to prescribe antibiotic prophylaxis for diabetics undergoing dental osseous surgery [31]. There is less evidence concerning the necessity of AP to prevent SSI following tooth extractions in diabetic patients. In a prospective observational study, Krishnan et al. [32] found a slight, but not significant increase in the risk of postoperative infections in type 2 diabetic patients undergoing tooth extractions with no AP, compared to healthy patients; other factors including glycemia, glycated haemoglobin, age and number of extractions were not associated with an increased risk of infection. Similar results were observed for insulin-dependent diabetic patients following tooth extraction without AP, with a small, though not statistically significant, increase in wound infections compared to healthy patients [33]. Based on the current evidence, the routine use of antibiotics in diabetic patients after single or multiple extractions does not seem to be justified, though a closer postoperative observation is required for these patients. Nevertheless, in the case presented, only $26 \%$ of respondents would not prescribe an AP.

Pericoronitis and periodontal abscess are common conditions that rarely present with systemic signs and symptoms. In the pericoronitis case (Q10), 79.3\% of the participants chose local antiseptics and anti-inflammatories with re-evaluation after 3 days. In Q11, most respondents $(46.2 \%)$ agreed on performing drainage and debridement of the pocket associated with postoperative antibiotics for 5 days, whereas $27.8 \%$ would only perform drainage and instrumentation, and $22.5 \%$ would perform antibiotic therapy before drainage and instrumentation. Among the free answers, some indicated the use of topical antibiotics after the socket debridement. The Clinical Practice Guideline by the European Federation of Periodontology [34] does not recommend the routine use of systemic antibiotics as an adjunct to subgingival debridement in patients with periodontitis, though periodontal abscesses are not specifically discussed. It was reported that the use of an antibiotic may be indicated when drainage/debridement is not possible and if the infection can spread and there are signs and symptoms of systemic involvement such as fever, lymphadenopathy, trismus etc. [35,36]. According to literature indications, the use of antibiotics in the case presented seems unjustified, since both the cause (subgingival plaque and calculus) and the effect (abscess) can be treated by drainage and instrumentation. However, antibiotics can be used as an adjuvant therapy in addition to the mechanical therapy in the case of severe infections, or as an attack therapy followed by the mechanical therapy when symptoms decrease after 2-3 days.

Q12 proposes the case of a non-surgical extraction in a non-oncological patient (n-OP) who regularly takes oral bisphosphonates (BP). In accordance with current guidelines, most respondents agreed on the need for adequate AP against aerobic and anaerobic pathogens $[37,38]$. Osteonecrosis of the jaws (ONJ) following tooth extraction is relatively rare among patients that take oral BP. On the contrary, dental extractions can be a precipitating factor for ONJ in $52-61 \%$ of patients taking intravenous BP. The case of patients under BP therapy is an exemplary situation in which, despite the low risk of the complication, $\mathrm{AP}$ is recommended due to the severity of the complication itself. To achieve a greater broad-spectrum action, a combination of amoxicillin $1 \mathrm{~g}$ t.i.d. and metronidazole $500 \mathrm{mg}$ t.i.d. has been recommended, although no definitive data are available indicating the optimal timing, preoperative, postoperative or both.

It is interesting that most respondents considered scarce oral hygiene as an indication for postoperative AP (C1), and that an oral hygiene session is not always performed before surgical procedures (C2). This means that many prescriptions could be avoided by performing routine oral hygiene procedures and instructing the patients before surgical interventions. Though it is good practice and common sense to perform an oral hygiene session in the case of plaque and calculus deposits, no study has investigated the relation 
between oral hygiene and postoperative infections in dentistry, and there is no evidence that antibiotics can reduce the risk of infection in cases of low compliance and scarce oral hygiene. Similarly, since the use of hemostatic materials or sutures (C3) in non-surgical extractions does not increase the risk of SSI [12], the use of antibiotics is not justified, as indicated by $82.2 \%$ of the participants. Conversely, in the case of ridge preservation procedures with bone grafting $(\mathrm{C} 4), 83.4 \%$ of participants agreed on the need for antibiotics, though literature data are scarce and conflicting on this issue [39,40], and it has been suggested that a single preoperative dose ( 2 or $3 \mathrm{~g}$ of amoxicillin) would be sufficient to prevent SSI after bone regeneration procedures [41].

The use of antibiotics after the extraction of teeth with periapical lesions is controversial. A systematic review has observed a high survival rate of implants placed in post-extraction sites of teeth with periapical/periodontal lesions after an adequate chemical-mechanical disinfection of the alveolus, whereas the effectiveness of local or systemic antibiotics is not proven [42].

Notably, $54.4 \%$ of the participants reported to have prescribed antibiotics they did not consider necessary (C7). Most respondents prescribed unnecessary antibiotics as a defensive medicine (options a, c, f). Interestingly, 11 of the 94 respondents indicated an insistence from colleagues (tutor, employer) or other specialists (cardiologist, general practitioner).

Finally, the participants reported that in the last 2 years they had attended courses on the correct use of antibiotics in dentistry ( $74.6 \%$ in C 8$)$, and that they were sufficiently informed about AMR (66.9\% in C9). Significantly, according to the participants, the prescriptive inappropriateness has medium to extreme diffusion (94.6\% in C10).

The results of this study, on the whole, confirm that the choice to prescribe an antibiotic is often based on subjective judgements and the biases of dentists. Several dentists prescribe unnecessary antibiotics as a defensive medicine or due to an overestimation of the risk and severity of postoperative complications. This can only be partially justified by the lack of guidelines, since some evidence already exists supporting a more reasonable use of antibiotics. These data are useful for identifying the cases in which the need for AP is still controversial, and could provide the starting point for clinical studies investigating the real usefulness of antibiotics for specific dental procedures.

This study has some limitations. Firstly, there was a relatively limited number of participants. Although the characteristics of the respondents indicate an adequate representativeness of the sample with respect to the general population of dental practitioners, the small number may limit the ability to generalize of the results. Furthermore, the study design and spread modality did not allow the characteristics of non-respondents to be identified. Finally, the limited number of answer options to the cases presented could have excluded other possible approaches of clinicians.

\section{Materials and Methods}

A survey was conducted among a non-random sample of Italian dentists through the administration of an anonymous questionnaire. The questionnaire (see Supplementary Materials) was prepared on the Google Forms platform and shared on different Italian dental social media and dental associations from June to December 2020. Considering the success and spread of new social media among dental clinicians, it was decided to share the questionnaire through these platforms in order to reach the highest number of participants from all regions, including both general practitioners and specialists. Recording the number of members of social media groups and dental associations at the start of the survey, a total of 2500 recipients was calculated. The opening page of the questionnaire was meant to provide information about the survey's intentions and the anonymous nature of the study. At that point, the recipients could leave or give their consent to continue with the questionnaire. The survey was structured in three parts. In the first section, demographic data were collected, including age, gender, education, professional experience and region. In the second section (Figure 2), clinical scenarios were presented, including the most 
common surgical procedures performed by dentists, and participants were asked to choose the most suitable AP or therapy regimen among those proposed. Cases of single or multiple non-surgical extractions were proposed in Q1, 2, 3, 4, 5, 6, 9 and 12. Q7 and Q8 reported the surgical extraction of an impacted wisdom tooth and the case of a single implant placement, respectively. Q10 and Q11 reported cases of pericoronitis and periodontal abscess, respectively, both without systemic signs and symptoms, and Q12 proposed the case of a non-surgical extraction in a non-oncological patient (n-OP) who regularly takes oral bisphosphonates (BP). Clinical variables included the patients' age, systemic conditions, and the type/number of surgical procedures. The standard response options for most questions (except in Q10, 11 and 12) included four possible timings and posology of broad-spectrum antibiotics, or the possibility of "no antibiotics". Short-form considerations (C1-10) about the use of antibiotics in specific procedures, and about AMR awareness, were also presented. For two questions (Q11 and C7) there was an opportunity for respondents to write a free answer.

The answers received have been assessed through the Google Forms percentage report. Descriptive statistics, including mean and percentage, were calculated for each variable.

\section{Conclusions}

The results of this survey indicate that the choice to prescribe prophylactic antibiotics to prevent postoperative complications after oral surgical procedures is mostly based on the subjective considerations of clinicians. Considering the increasing phenomena of antimicrobial resistance, it is essential that clinicians prescribe prophylactic antibiotics only when strictly necessary, since many prescriptions could be avoided based on the current evidence. On the other hand, scientific societies should elaborate specific guidelines for the correct use of prophylactic antibiotics to prevent SSIs, specifically addressing the most controversial issues. To this end, more randomized controlled trials investigating the need of antibiotics prophylaxis for the most common oral surgical procedures are urgently required.

Supplementary Materials: The following Supplementary Materials are available online at https: / /www.mdpi.com/article/10.3390/antibiotics10080949/s1, PDF file: Questionnaire.

Author Contributions: Conceptualization, M.L. and G.P.; methodology, G.P., G.S. and G.M.; investigation, M.L. and G.P.; data curation, G.P.; writing-original draft preparation, M.L. and G.P.; writing-review and editing, M.L. and G.P.; supervision, A.D.B. and L.L. All authors have read and agreed to the published version of the manuscript.

Funding: This research received no external funding.

Institutional Review Board Statement: Not applicable.

Informed Consent Statement: Not applicable.

Data Availability Statement: The data presented in this study are available on request from the corresponding author.

Conflicts of Interest: The authors declare no conflict of interest.

\section{References}

1. Oberoi, S.S.; Dhingra, C.; Sharma, G.; Sardana, D. Antibiotics in dental practice: How justified are we. Int. Dent. J. 2015, 65, 4-10. [CrossRef] [PubMed]

2. D'Agostino, S.; Dolci, M. Antibiotic therapy in oral surgery: A cross sectional survey among Italian dentists. J. Biol. Regul. Homeost. Agents. 2020, 34, 1549-1552.

3. Tebano, G.; Dyar, O.J.; Beovic, B.; Béraud, G.; Thilly, N.; Pulcini, C. ESCMID Study Group for Antimicrobial stewardship (ESGAP). Defensive medicine among antibiotic stewards: The international ESCMID AntibioLegalMap survey. J. Antimicrob. Chemother. 2018, 73, 1989-1996. [CrossRef]

4. European Centre for Disease Prevention and Control. Antimicrobial Resistance in the EU/EEA (EARS-Net)-Annual Epidemiological Report 2019; ECDC: Stockholm, Sweden, 2020. 
5. $\quad$ Bratzler, D.W.; Dellinger, E.P.; Olsen, K.M.; Perl, T.M.; Auwaerter, P.G.; Bolon, M.K.; Fish, D.N.; Napolitano, L.M.; Sawyer, R.G.; Slain, D.; et al. Clinical practice guidelines for antimicrobial prophylaxis in surgery. Am. J. Health Syst. Pharm. 2013, 70, 195-283. [CrossRef] [PubMed]

6. Enzler, M.J.; Berbari, E.; Osmon, D.R. Antimicrobial prophylaxis in adults. Mayo Clin. Proc. 2011, 86, 686-701. [CrossRef] [PubMed]

7. Thompson, W.; Williams, D.; Pulcini, C.; Sanderson, S.; Calfon, P.; Verma, M. The Essential Role of the Dental Team in Reducing Antibiotic Resistance; FDI World Dental Federation: Geneva, Italy, 2020.

8. The Medicines Utilisation Monitoring Centre. National Report on Antibiotics Use in Italy; Italian Medicines Agency: Rome, Italy, 2020.

9. Della Polla, G.; Bianco, A.; Mazzea, S.; Napolitano, F.; Angelillo, I.F. Preoperative Antibiotic Prophylaxis in Elective Minor Surgical Procedures among Adults in Southern Italy. Antibiotics 2020, 9, 713. [CrossRef]

10. Walsh, L.J.; Ford, P.J.; McGuire, T.; van Driel, M.; Hollingworth, S.A. Trends in Australian dental prescribing of antibiotics: 2005-2016. Aust Dent. J. 2021, 23, 1-5.

11. Carlet, J.; Collignon, P.; Goldmann, D.; Goossens, H.; Gyssens, I.C.; Harbarth, S.; Jarlier, V.; Levy, S.B.; N’Doye, B.; Pittet, D.; et al Society's failure to protect a precious resource: Antibiotics. Lancet 2011, 378, 369-371. [CrossRef]

12. Barone, A.; Chatelain, S.; Derchi, G.; Di Spirito, F.; Martuscelli, R.; Porzio, M.; Sbordone, L. Antibiotic's effectiveness after erupted tooth extractions: A retrospective study. Oral Dis. 2020, 26, 967-973. [CrossRef]

13. Cinquini, C.; Marchionni, S.; Derchi, G.; Miccoli, M.; Gabriele, M.; Barone, A. Non-impacted tooth extractions and antibiotic treatment: A RCT study. Oral Dis. 2021, 27, 1042-1051. [CrossRef]

14. Wilson, W.; Taubert, K.A.; Gewitz, M.; Lockhart, P.B.; Baddour, L.M.; Levison, M.; Bolger, A.; Cabell, C.H.; Takahashi, M.; Baltimore, R.S. Prevention of infective endocarditis: Guidelines from the American Heart Association: A guideline from the American Heart Association Rheumatic Fever, Endocarditis, and Kawasaki Disease Committee, Council on Cardiovascular Disease in the Young, and the Council on Clinical Cardiology, Council on Cardiovascular Surgery and Anesthesia, and the Quality of Care and Outcomes Research Interdisciplinary Working Group. Circulation 2007, 116, 1736-1754. [PubMed]

15. Thornhill, M.H.; Gibson, T.B.; Cutler, E.; Dayer, M.J.; Chu, V.H.; Lockhart, P.B.; O'Gara, P.T.; Baddour, L.M. Antibiotic Prophylaxis and Incidence of Endocarditis Before and After the 2007 AHA Recommendations. J. Am. Coll. Cardiol. 2018, 72, $2443-2454$. [CrossRef] [PubMed]

16. Suda, K.J.; Calip, G.S.; Zhou, J.; Rowan, S.; Gross, A.E.; Hershow, R.C.; Perez, R.I.; McGregor, J.C.; Evans, C.T. Assessment of the Appropriateness of Antibiotic Prescriptions for Infection Prophylaxis Before Dental Procedures, 2011 to 2015. JAMA Netw. Open. 2019, 2, e193909. [CrossRef]

17. Arteagoitia, I.; Ramos, E.; Santamaria, G.; Barbier, L.; Alvarez, J.; Santamaria, J. Amoxicillin/clavulanic acid $2000 / 125$ mg to prevent complications due to infection following completely bone-impacted lower third molar removal: A clinical trial. Oral Surg. Oral Med. Oral Pathol. Oral Radiol. 2015, 119, 8-16. [CrossRef]

18. Kamolratanakul, P.; Jansisyanont, P. A review of antibiotic prophylaxis protocols in oral and maxillofacial surgery. J. Oral Maxillofac. Surg. Med. Pathol. 2018, 30, 395-404. [CrossRef]

19. Lodi, G.; Azzi, L.; Varoni, E.M.; Pentenero, M.; Del Fabbro, M.; Carrassi, A.; Sardella, A.; Manfredi, M. Antibiotics to prevent complications following tooth extractions. Cochrane Database Syst Rev. 2021, 2, CD003811.

20. Sancho-Puchades, M.; Herráez-Vilas, J.M.; Berini-Aytés, L.; Gay-Escoda, C. Antibiotic prophylaxis to prevent local infection in Oral Surgery: Use or abuse? Med. Oral Patol. Oral Cir. Bucal. 2009, 14, E28-E33. [PubMed]

21. Romandini, M.; De Tullio, I.; Congedi, F.; Kalemaj, Z.; D’Ambrosio, M.; Laforí, A.; Quaranta, C.; Buti, J.; Perfetti, G. Antibiotic prophylaxis at dental implant placement: Which is the best protocol? A systematic review and network meta-analysis. J. Clin. Periodontol. 2019, 46, 382-395. [CrossRef]

22. Esposito, M.; Grusovin, M.G.; Worthington, H.V. Interventions for replacing missing teeth: Antibiotics at dental implant placement to prevent complications. Cochrane Database Syst. Rev. 2013, 7, CD004152. [CrossRef]

23. Rodríguez Sánchez, F.; Rodríguez Andrés, C.; Arteagoitia, I. Which antibiotic regimen prevents implant failure or infection after dental implant surgery? A systematic review and meta-analysis. J. Craniomaxillofac. Surg. 2018, 46, 722-736. [CrossRef] [PubMed]

24. Rodríguez Sánchez, F.; Arteagoitia, I.; Rodríguez Andrés, C.; Caiazzo, A. Antibiotic prophylaxis habits in oral implant surgery among dentists in Italy: A cross-sectional survey. BMC Oral Health. 2019, 19, 265. [CrossRef]

25. Rodríguez Sánchez, F.; Arteagoitia, I.; Rodríguez Andrés, C.; Bruers, J. Antibiotic prophylaxis prescribing habits in oral implant surgery in the Netherlands: A cross-sectional survey. BMC Oral Health. 2019, 19, 281. [CrossRef]

26. Arteagoitia, I.; Rodríguez-Andrés, C.; Rodríguez-Sánchez, F. Antibiotic prophylaxis habits in dental implant surgery among dentists in Spain. A cross-sectional survey. Med. Oral Patol. Oral Cir. Bucal. 2018, 23, e608-e618. [CrossRef]

27. Lund, B.; Hultin, M.; Tranaeus, S.; Naimi-Akbar, A.; Klinge, B. Complex systematic review-Perioperative antibiotics in conjunction with dental implant placement. Clin. Oral Implants Res. 2015, 26 (Suppl. S11), 1-14. [CrossRef]

28. Mazzocchi, A.; Passi, L.; Moretti, R. Retrospective analysis of 736 implants inserted without antibiotic therapy. J. Oral Maxillofac. Surg. 2007, 65, 2321-2323. [CrossRef] [PubMed]

29. Bernabeu-Mira, J.C.; Peñarrocha-Diago, M.; Peñarrocha-Oltra, D. Prescription of Antibiotic Prophylaxis for Dental Implant Surgery in Healthy Patients: A Systematic Review of Survey-Based Studies. Front Pharmacol. 2021, 11, 588333. [CrossRef] 
30. Martin, E.T.; Kaye, K.S.; Knott, C.; Nguyen, H.; Santarossa, M.; Evans, R.; Bertran, E.; Jaber, L. Diabetes and Risk of Surgical Site Infection: A Systematic Review and Meta-analysis. Infect. Control Hosp. Epidemiol. 2016, 37, 88-99. [CrossRef] [PubMed]

31. Barasch, A.; Safford, M.M.; Litaker, M.S.; Gilbert, G.H. Risk factors for oral postoperative infection in patients with diabetes. Spec. Care Dentist 2008, 28, 159-166. [CrossRef]

32. Krishnan, B.; Prasad, G.A.; Saravanan, R.; Madhan, B.; Kadhiravan, T. Do preoperative glycosylated hemoglobin (HbA1C) and random blood glucose levels predict wound healing complications following exodontia in type 2 diabetes mellitus patients?-a prospective observational study. Clin. Oral Investig. 2021, 25, 179-185. [CrossRef]

33. Power, D.J.; Sambrook, P.J.; Goss, A.N. The healing of dental extraction sockets in insulin-dependent diabetic patients: A prospective controlled observational study. Aust. Dent. J. 2019, 64, 111-116. [CrossRef] [PubMed]

34. Sanz, M.; Herrera, D.; Kebschull, M.; Chapple, I.; Jepsen, S.; Beglundh, T.; Sculean, A.; Tonetti, M.S.; EFP Workshop Participants and Methodological Consultants. Treatment of stage I-III periodontitis-The EFP S3 level clinical practice guideline. J. Clin. Periodontol. 2020, 47, 4-60. [CrossRef]

35. Dar-Odeh, N.S.; Abu-Hammad, O.A.; Al-Omiri, M.K.; Khraisat, A.S.; Shehabi, A.A. Antibiotic prescribing practices by dentists: A review. Ther. Clin. Risk Manag. 2010, 6, 301-306. [CrossRef] [PubMed]

36. Segura-Egea, J.J.; Gould, K.; Şen, B.H.; Jonasson, P.; Cotti, E.; Mazzoni, A.; Sunay, H.; Tjäderhane, L.; Dummer, P.M.H. European Society of Endodontology position statement: The use of antibiotics in endodontics. Int. Endod. J. 2018, 51, 20-25. [CrossRef]

37. Yarom, N.; Shapiro, C.L.; Peterson, D.E.; Van Poznak, C.H.; Bohlke, K.; Ruggiero, S.L.; Migliorati, C.A.; Khan, A.; Morrison, A.; Anderson, H.; et al. Medication-Related Osteonecrosis of the Jaw: MASCC/ISOO/ASCO Clinical Practice Guideline. J. Clin. Oncol. 2019, 37, 2270-2290. [CrossRef]

38. Campisi, G.; Mauceri, R.; Bertoldo, F.; Bettini, G.; Biasotto, M.; Colella, G.; Consolo, U.; Di Fede, O.; Favia, G.; Fusco, V.; et al. Medication-Related Osteonecrosis of Jaws (MRONJ) Prevention and Diagnosis: Italian Consensus Update 2020. Int. J. Environ. Res. Public Health 2020, 17, 5998. [CrossRef]

39. Khouly, I.; Braun, R.S.; Silvestre, T.; Musa, W.; Miron, R.J.; Demyati, A. Efficacy of antibiotic prophylaxis in intraoral bone grafting procedures: A systematic review and meta-analysis. Int. J. Oral Maxillofac. Surg. 2020, 49, 250-263. [CrossRef] [PubMed]

40. Klinge, A.; Khalil, D.; Klinge, B.; Lund, B.; Naimi-Akbar, A.; Tranaeus, S.; Hultin, M. Prophylactic antibiotics for staged bone augmentation in implant dentistry. Acta Odontol. Scand. 2020, 78, 64-73. [CrossRef]

41. Salgado-Peralvo, A.O.; Mateos-Moreno, M.V.; Velasco-Ortega, E.; Peña-Cardelles, J.F.; Kewalramani, N. Preventive antibiotic therapy in bone augmentation procedures in oral implantology: A systematic review. J. Stomatol. Oral Maxillofac. Surg. 2021, 22, S2468-S7855.

42. Chrcanovic, B.R.; Martins, M.D.; Wennerberg, A. Immediate placement of implants into infected sites: A systematic review. Clin Implant Dent. Relat. Res. 2015, 17 (Suppl. S1), e1-e16. [CrossRef] [PubMed] 